\title{
Specificity of Loxosceles $\alpha$ clade phospholipase D enzymes for choline- containing lipids: role of a conserved aromatic cage
}

\author{
Short title: Role of an aromatic cage for PLD lipid specificity
}

Emmanuel E. Moutoussamy ${ }^{1,2}$, Qaiser Waheed ${ }^{1,2}$, PhD, Greta J. Binford ${ }^{3}$, PhD, Hanif M.Khan ${ }^{1,2}$, PhD, Shane M. Moran ${ }^{4}$, Anna R. Eitel ${ }^{4}$, Matthew H.J. Cordes ${ }^{4}$, PhD, Nathalie Reuter ${ }^{* 1,5}$, PhD

${ }^{1}$ Computational Biology Unit, Department of Informatics, University of Bergen, Bergen, Norway
${ }^{2}$ Department of Biological Sciences, University of Bergen, Bergen, Norway
${ }^{3}$ Department of Biology, Lewis and Clark College, Portland, Oregon, United States
${ }^{4}$ Department of Chemistry and Biochemistry, University of Arizona, Arizona, United States
${ }^{5}$ Department of Chemistry, University of Bergen, Bergen, Norway

*Corresponding author: Nathalie Reuter, E-mail: nathalie.reuter@uib.no

Present addresses:

Hanif Khan: Centre for Molecular Simulation, Department of Biological Sciences, University of Calgary, Calgary, Alberta, Canada

Qaiser Waheed: Department of Chemistry, Bioscience and Environmental Engineering, University of Stavanger, Norway

\section{ABSTRACT}

Spider venom GDPD-like phospholipases D ( SicTox) have been identified to be one of the major toxins in recluse spider venom. They are divided into two major clades: the $\alpha$ clade and the $\beta$ clade. Most $\alpha$ clade toxins present high activity against lipids with choline head groups such as sphingomyelin, while activities in $\beta$ clade toxins vary and include preference for substrates containing ethanolamine headgroups (Sicarius terrosus, St_ßIB1). A structural comparison of available PLDs structures reveals a conserved aromatic cage in the $\alpha$ clade. To test the potential influence of the aromatic cage on membrane-lipid specificity we performed molecular-dynamics (MD) simulations of the binding of several PLDs onto lipid bilayers containing choline headgroups; two SicTox from the $\alpha$ clade, Loxosceles intermedia $\alpha \mathrm{IA} 1$

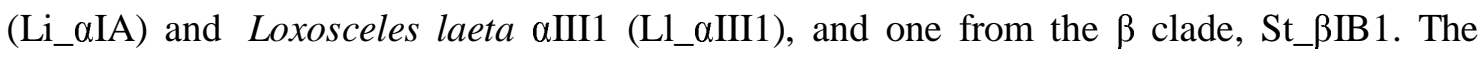
simulation results reveal that the aromatic cage captures a choline-headgroup and suggest that the cage plays a major role in lipid specificity. We also simulated an engineered St_ßIB1, 
where we introduced the aromatic cage, and this led to binding with choline-containing lipids. Moreover, a multiple sequence alignment revealed the conservation of the aromatic cage among the $\alpha$ clade PLDs. Here, we confirmed the membrane binding site of $\alpha$ and $\beta$ clade PLDs on choline and ethanolamine-containing bilayers, respectively. Furthermore, our results suggest a major role in choline lipid recognition of the aromatic cage of the $\alpha$ clade PLDs. The MD simulation results are supported by in vitro liposome binding assay experiments.

\section{KEYWORDS}

Sphingomyelinase D, phospholipase D, SicTox, Loxoscelism, peripheral membrane enzymes, toxin, molecular dynamics simulation, aromatic cage

\section{INTRODUCTION}

Sphingomyelinase D/phospholipase D (SMaseD/PLD) enzymes are one of the major toxins in venom of spiders in the family Sicariidae (1-3) including Loxosceles, the recluse spiders. Sicariid spiders also include six-eyed sand spiders in the genera Sicarius and Hexopthalma (4). The gene family comprising the sicariid SMaseD/PLD toxins is called SicTox reflecting evidence that this venom toxin is a synapomorphy for the family Sicariidae and is not a homolog of other PLDs (5). Envenomation by recluse spiders causes necrotic tissue breakdown in mammals, a condition known as loxoscelism, and leads to skin degeneration and systemic reactions in the worst cases (2). SicTox enzymes are sufficient causative agents for this syndrome in mammals (6) and thus are pharmaceutical targets $(7,8)$. While sicariid spiders are found natively on all continents except Australia, treatments of loxoscelism are typically antibody based and available in some regions of the Americas (9). 
SicTox enzymes catalyse the cleavage of lipid headgroups forming an alcohol and a cyclic phospholipid (10). The structures of two Loxosceles PLD have been resolved by X-ray (Figure 1): Loxosceles laeta L1_aIII1i (PDB ids: 1XX1 (11), 2F9R (11)) and Loxosceles intermedia Li-aIA1 (PDB id: 3RLH (12)). Their fold consists of a distorted TIM-barrel, a domain also found in many other phospholipases. Their membrane-binding region is called the $\mathrm{i}$-face (for interfacial face) and is thought to consist of the loops $\beta 2 \alpha 2$ (also called catalytic loop), $\beta 5 \alpha 5$ and $\beta 6 \alpha 6$ (flexible loop) (11), all shown on Figure 1.

The Loxosceles SMaseD/PLD enzymes are classified in either the $\alpha$ clade or the $\beta$ clade (13). Members of the $\alpha$-clade have high catalytic activity against sphingomyelin (SM), while activity in the $\beta$ clade is more variable (14-19). Only $\beta$ clade enzymes have been found in Sicarius and Hexopthalma venoms while Loxosceles contain both $\alpha$ and $\beta$ clade SicTox toxins paralogs. Using ${ }^{31} \mathrm{P}$ NMR, Lajoie et al. recently showed that a $\beta$ clade enzyme from Sicarius terrosus (St_ßIB1) had a strong preference for substrates with ethanolamine headgroups vs. choline-containing lipids (14) in detergent micelles containing the lipid substrates. Lajoie et al. also measured the activity of an $\alpha$ and a $\beta$ clade enzyme from

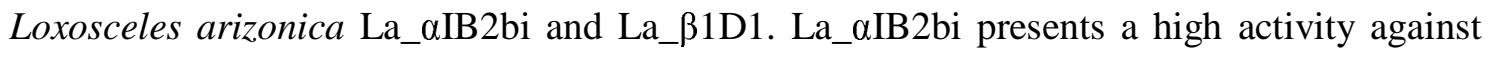
choline-containing lipids and interestingly La_ßID1 from the $\beta$ clade shows little discrimination between ethanolamine and choline-containing substrates. The substrate specificity of the SMases is thought to be related to the relative abundance of ethanolaminecontaining lipids in the cell membranes of arthropods (19), which are preferred prey of sicariid spiders (20). Lajoie et al. also resolved the X-ray structure of St_ßIB1 (PDB ID 4Q6X, Fig.1); it resembles the structures of the $\alpha$ clade SicTox enzymes from Loxosceles and there are only subtle differences in is the active or ligand binding sites. This led the authors to suggest that the i-face of the SicTox SMases/PLDs is likely to play a role in specific lipid recognition. 
While the three previously mentioned loops are thought to constitute the i-face, there has not been any experimental investigation to identify which particular amino acids are involved in membrane binding. As demonstrated by many examples, molecular simulations have proven to be a useful tool to predict interfacial binding sites (IBS) of peripheral membrane proteins and map protein-lipid interactions (21-25). Of particular relevance are our efforts at characterizing the membrane binding site of a bacterial phosphatidylinositolspecific phospholipase C from Bacillus thurigensis (BtPI-PLC) $(21,25,26)$. BtPI-PLC binds preferentially to PC-rich lipid bilayers, is folded as a distorted $\alpha \beta$ TIM barrel, and just as for the SicTox SMases/PLDs the BtPI-PLC IBS consists of a few loops located on the same side of the $\alpha \beta$-barrel as the active site. Using a combination of MD simulations and in vitro $\mathrm{K}_{\mathrm{d}}$ determination of the wild type enzyme and mutants, we could show that the preference of BtPI-PLC for PC lipids was the result of cation- $\pi$ interactions between surface-exposed tyrosine residues and the choline groups of PC lipids $(21,25-27)$ and that those cation- $\pi$ interactions between choline and interfacial tyrosine could contribute up to 2 to $3 \mathrm{kcal} / \mathrm{mol}$ to the protein-membrane affinity (28).

Closely observing the amino acid sequences and available X-ray structures of the

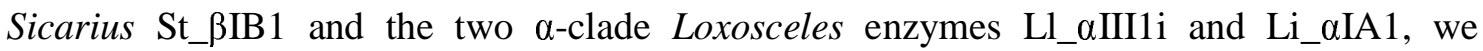
noticed that the i-face of the $\alpha$-clade enzymes is enriched in tyrosine and tryptophan residues. Li- $\alpha$ IA1 counts three tyrosines on the catalytic loop (Y44, Y46, Y60), L1_aIII1 has two tyrosines (Y44, Y46, Y62) and a tryptophan (W60), while St_ßIB1 has only one tyrosine on this loop (Y44). In addition, Li- $\alpha$ IA1 has a tyrosine (Y98) on the $\beta 3 \alpha 3$ loop and Ll_ $\alpha I I I 1 i$ has a tyrosine (Y169) on the $\beta 5 \alpha 5$. Interestingly, the catalytic loop of La_ $\alpha$ IB2bi also has three tyrosines and a tryptophan at positions equivalent to Y44, Y46, Y62 and W60 in Ll- $\alpha$ III1i, according to a sequence alignment (14). The position and orientation of these conserved

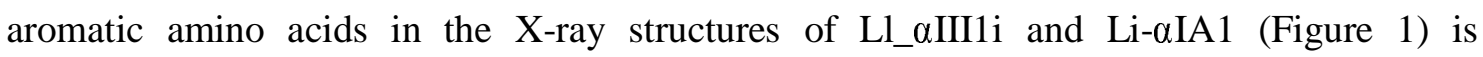


compatible with the formation of an aromatic cage as observed in proteins binding cholinecontaining ligands $(29)(27,30)(31)$. We pose the hypothesis that the tyrosine and tryptophan residues on the $\mathrm{i}$-face of La_ $\alpha \mathrm{IB} 2 \mathrm{bi}$ and other $\alpha$-clade enzymes provides a mechanism to selectively recognize choline-containing lipids as ligands.

In this work, we evaluate this hypothesis using MD simulations and liposome binding assay experiments. As there is no X-ray structure of La_aIB2bi we chose to simulate

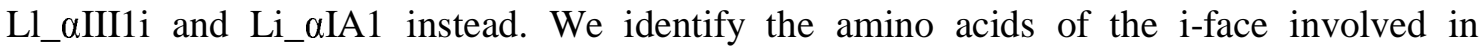
membrane binding by characterizing their interactions with lipid bilayers containing either only palmitoyl-oleyl-phosphatidyl choline lipids (POPC) or a mixture of POPC, sphingomyelin (SM) and cholesterol (CHOL). We also seek to understand how St_ßIB1 interacts with phosphatidylethanolamine (PE) lipids and performed simulations of the $\beta$ clade enzyme with bilayers consisting of both PC and PE lipids. Finally, we introduced aromatic amino acids in selected positions of the $\beta$ clade enzyme to evaluate if it then acquires affinity towards choline-containing bilayers. We also performed in vitro liposome binding assay to

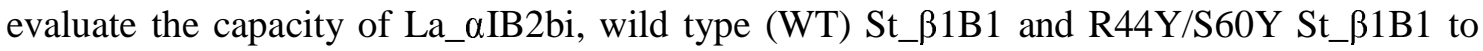
bind to SM:CHOL (1:1) liposomes.

\section{FIGURE 1 HERE}

\section{METHODS}

\section{Dataset for structural and multiple sequence alignment}

We retrieved X-ray structures from the Protein Data Bank (PDB) (32) for Li_aIA1

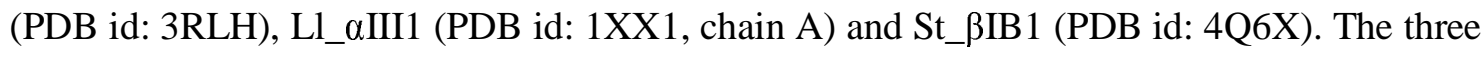


structures were aligned with MUSTANG (v 3.2.3) (33). Clustal Omega (34) was used to align multiple sequences alignment. Default parameters were used. We retrieved the sequences of 25 PLDs representatives of subgroups in the two clades (12 $\alpha$ - and $6 \beta$-clade

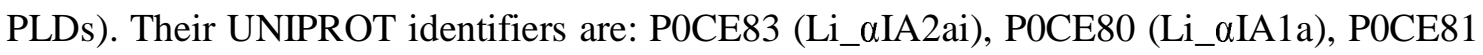

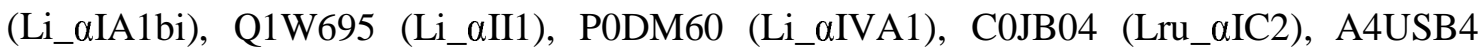

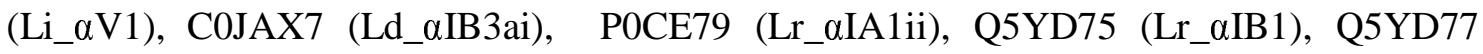

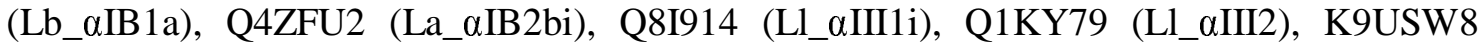

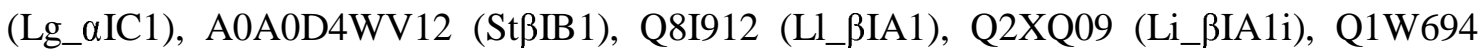

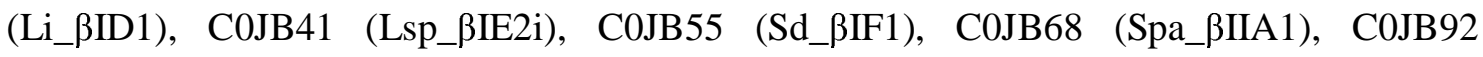

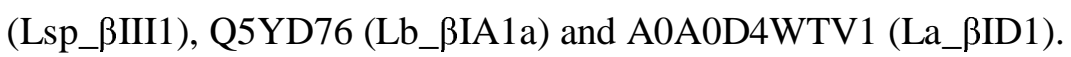

\section{System preparation}

Lipid bilayers. Bilayers consisting of 256 lipid molecules (128 lipids in each leaflet) were built with the CHARMM-GUI (35) using the Membrane Builder module (36-38). POPC and POPC:POPE (50:50) bilayers were simulated for $200 \mathrm{~ns}$ (NPT) while the POPC:PSM:CHOL (70:20:10) bilayer was simulated for 500 ns (NPT) using the CHARMM36 force field (39-41) The calculated area per lipid for POPC is $65.0 \AA^{2}$ in agreement with earlier simulations using the same force field $\left(65.5 \AA^{2}\right)(22,39)$. The experimental value reported by Kuc $\square$ erka is $68.5 \AA^{2}$ (42). The addition of POPE to construct a POPC:POPE (50:50) bilayer decreases the calculated area per lipid to $61.8 \AA^{2}$, in good agreement with the $59.4 \AA^{2}$ from recent simulation data (43). As expected (44), the addition of PSM and CHOL to POPC also reduces the area per lipid, measured to be $52.5 \AA^{2}$ for the POPC:PSM:CHOL (70:20:10) mixture.

Protein-bilayer systems. We set up eight protein-bilayer systems: Li_aIA1 and

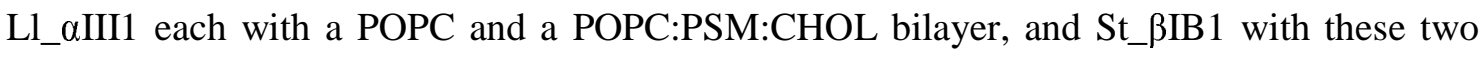


bilayers plus a POPC:POPE bilayer. In addition a mutant of St_ßIB1 was prepared with a POPC bilayer; two amino acids (R44 and S60) were replaced by tyrosines using UCSF Chimera (45). Protein structures were placed slightly above the surface of the relevant bilayers at a distance of $48 \AA$ between the center of mass of bilayer and the center of mass of the protein. The protein were positioned in the binding orientation predicted by OPM (46) for

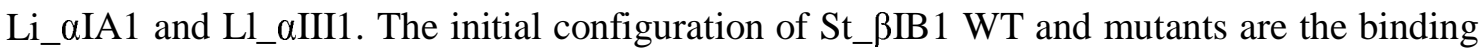
orientation predicted by the PPM server (46). The systems were solvated and neutralized using VMD (v 1.9.1) (47). The composition of the eight simulated systems, included number of lipids, water molecules and ions, is reported in Table S1.

\section{Molecular dynamics simulations}

All simulations were performed using NAMD (v 2.13) (48) with the CHARMM36 force field (39-41) and its CHARMM-WYF extension for the treatment of aromatics-choline interactions $(49,50)$. The systems were first subjected to energy minimization with conjugate gradients (10000 steps) and an equilibration of $2 \mathrm{~ns}$ in the NPT ensemble. Next the production runs were run in the same ensemble for $300 \mathrm{~ns}$ using the last coordinates and velocities of the equilibration step. All the steps were done with an integration step of $2 \mathrm{fs}$. We ran two replicates for each of the systems listed in Table S1; replicas started from the same minimization step but differ by the equilibration step and therefore, different velocity distributions were used for the production run. Before equilibration and production runs, the mutant was subjected to extra equilibration in water for $1 \mathrm{~ns}$. The temperature was set to 310 $\mathrm{K}$ and controlled using Langevin dynamics (temperature damping coefficient: 1.0). The pressure was set to 1 atm using the Langevin piston method with an oscillation period of 200 fs and a damping timescale of 50 fs. The SHAKE algorithm was applied in order to constraint all bonds between hydrogen and heavy atoms (51). Particle mesh Ewald was used for the 
treatment of long range electrostatic interactions (52).

\section{Trajectory analysis}

The analysis of the trajectories was done on the window 150-300 ns, since all proteins were bound to the bilayers and the protein RMSD was stable for all $150 \mathrm{~ns}$ into the production run (Fig. S2). Trajectory analysis was done using CHARMM (v33b1) (53) for the inventory of lipid-protein interactions, VMD (v 1.9.1) (47) for the electron density plots and MDAnalysis $(54,55)$ for the calculation of the depth of insertion. We analysed three types of interactions: hydrophobic contacts, hydrogen bonds and cation- $\pi$ interactions which we identified using the same definition as in Grauffel et al. (25). Briefly hydrophobic contacts are considered to exist if two non-bonded candidate atoms are within a distance of $3 \mathrm{~A} \square$. Candidate atoms are atoms in aliphatic groups of amino acids side chains (list provided in supporting information, Table S2). For hydrogen bonds the distance between the hydrogen and the donor of hydrogen bond atom should be below or equal to $2.4 \AA$, and the angle between the hydrogen bond donor, the hydrogen and the hydrogen bond acceptor should be higher than or equal to $130^{\circ}$. Cation $-\pi$ interactions between the aromatic rings of tyrosines and tryptophans were considered to exist when all distances between the aromatic carbon and nitrogen atoms and the choline nitrogen were below $7 \mathrm{~A} \square$. In addition, these distances should not differ by more than 1.5 $\mathrm{A} \square$. Interactions were considered only if the criteria were met for at least 10ps and if the interaction was observed in both replicates. The depth of insertion for a given amino acid is the distance between its $\mathrm{C} \alpha$ atom and the average upper phosphate plane. The position of the plane was calculated on the last frame of the simulation.

\section{Liposome binding assays}

T7 expression vectors (pHIS8) containing genes encoding La_ $\alpha$ IB2bi and St_ß1B1 were constructed previously $(56,57)$. The R44Y/S60Y variant of St_ß1B1 was constructed using 
QuikChange mutagenesis (Agilent, Santa Clara, California, USA) of the wild-type construct.

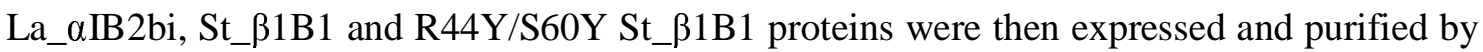
nickel affinity chromatography as described previously (10), followed by extensive dialysis into insect physiological saline (IPS; $5 \mathrm{mM}$ potassium phosphate [pH 6.5], $100 \mathrm{mM}$ potassium chloride, $4 \mathrm{mM}$ sodium chloride, $15 \mathrm{mM}$ magnesium chloride, $2 \mathrm{mM}$ calcium chloride). Thin lipid films were prepared by dissolving equimolar amounts of egg sphingomyelin and cholesterol to a total lipid concentration of $9 \mathrm{mg} / \mathrm{mL}$ in $2: 1(\mathrm{v} / \mathrm{v})$ chloroform:methanol, evaporating the solvent in a glass test tube with a weak stream of argon while rotating the tube, and drying the film under vacuum at $45{ }^{\circ} \mathrm{C}$ for at least $2 \mathrm{~h}$. Films were converted to liposomes (large multilamellar vesicles) by suspending them at $36 \mathrm{mg} / \mathrm{mL}$ lipid concentration in $10 \mathrm{mM}$ HEPES ( $\mathrm{pH} 7.5$ ), $150 \mathrm{mM}$ sodium chloride, followed by heating at $65{ }^{\circ} \mathrm{C}$ for $1 \mathrm{~h}$ with occasional vortexing. For the liposome binding assay, $1.5-2 \mu \mathrm{g}$ protein in $10 \mathrm{~mL}$ IPS was mixed with $10 \mathrm{~mL}$ of an $18 \mathrm{mg} / \mathrm{mL}$ liposome solution and incubated at $25{ }^{\circ} \mathrm{C}$ for $30 \mathrm{~min}$, followed by centrifugation for $10 \mathrm{~min}$ at ambient temperature at $16000 \mathrm{~g}$. Supernatant and pellet were separated, converted to gel samples of equal volume, loaded onto Tris-tricine SDS-PAGE gels, and electrophoresed for at least $45 \mathrm{~min}$ at $150 \mathrm{~V}$. Gels were stained with Coomassie Brilliant Blue R-250, destained, and photographed. Bands were quantified using ImageJ software (58). Values reported in the text are the average of three experiments; reported errors represent the standard error of the mean. 


\section{RESULTS}

\section{(1) Cation- $\pi$ interactions between the aromatic cage in $\alpha$ clade enzymes and POPC}

\section{FIGURE 2 HERE}

We performed molecular dynamics simulations of each of the three PLDs (Li_aIA1,

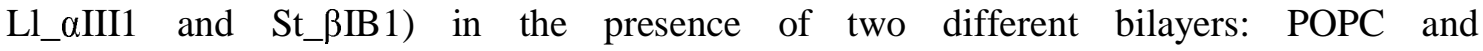
POPC:PSM:CHOL (70:20:10). Each simulation was initiated with the protein positioned slightly above the bilayer. The RMSD of the protein backbone with respect to structures after equilibration remains low during the production runs (Cf Figures S1-S2) and show deviations of at most $1.2 \AA$.

The $\beta$ clade Sicarius enzyme St_BIB1 does not bind to the PC-containing bilayers and diffuses away rapidly (Fig. 2 and 3A), indicating a low affinity for POPC lipids. The $\alpha$ clade

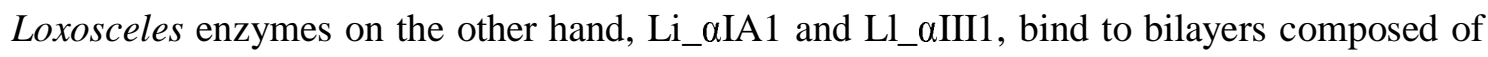
POPC (Fig. 2 and 3A) and POPC:SM:CHOL (Fig. 4A and S3) within 100 ns. Figure 4A shows the electron density profiles (EDP) and the depth of insertion below the phosphate planes calculated for each amino acid. The two enzymes are anchored rather superficially in the bilayer, irrespective of the type of bilayer, and only a few residues are inserted under the

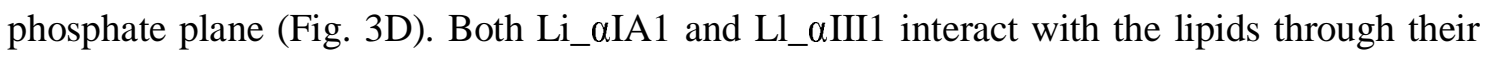
$\beta 2 \alpha 2$ catalytic loop while the other loops, including $\beta 6 \alpha 6$ remain higher above the interface (Fig. 3B-D). We observed hydrophobic contacts with the lipids chains for only a few

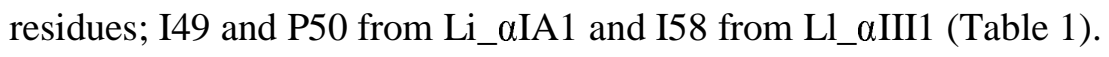

\section{FIGURE 3 AND TABLE 1 HERE}


Amino acids involved in hydrogen bonds, hydrophobic contacts and cation- $\pi$ interactions with the lipids are listed in Table 1. Numerous residues located in the catalytic loop establish a hydrogen bond network with the bilayer. Most occupancies are low $(<50 \%)$ and most hydrogen bonds are achieved by the side chains except for I49, C51, G54 in

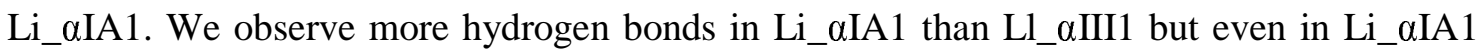
the occupancies of hydrogen bonds are not high except for K58 (above 70\%). The side chain of R59 in Ll_aIII1 also engages in long-lasting hydrogen bonds with the lipids (> 90\% occupancy). Noteworthy are the hydrogen bonds achieved by Y44, Y46, Y60 and Y98 in

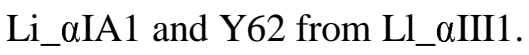

These tyrosines also establish cation- $\pi$ interactions with the choline groups of the lipid heads (Table 1 and Figure 4). In Li_aIA1 Y44, Y46 and Y60 form an aromatic cage occupied by a choline group. The occupancies of the cation- $\pi$ interaction between those tyrosines and the bilayer are between 29 and 46\%. In L1_aIII1, Y44, Y46, W60 form an equivalent cage and the occupancies of the cation- $\pi$ interactions are between 27.54 and $89 \%$. In the course of the $300 \mathrm{~ns}$ of simulation, the cage can be occupied by either the same lipid or by up to 3 subsequent lipids during the simulation (Fig. S4-S6). Moreover, the two $\alpha$ clade PLDs contain an additional tyrosine establishing cation- $\pi$ interactions with the bilayer: Y98 from the $\beta 3 \alpha 3$ loop in Li_aIA1 (46-60\% depending on the bilayer) and Y62 from the catalytic loop in Ll_aIII1 (39-46\%).

FIGURE 4 HERE

(2) Dense hydrogen bond network between aspartates in the $\beta$ clade enzyme and POPE

FIGURE 5 and TABLE 2 HERE 
Lajoie et al. showed that St_ßIB1 turns over ethanolamine-containing ceramides and lysophospholipids 2 to 3 orders of magnitude faster than corresponding choline-containing lipids. - The binding pocket for the lipid headgroup in PLDs has been identified using molecular docking and does not appear to account for the difference of lipid specificity. We ran simulations of $\mathrm{St} \_\beta \mathrm{IB} 1$ in the presence of a POPC:POPE (50:50) bilayer to predict the binding site of the Sicarius enzyme and analyse the protein-lipid interactions.

Unlike the simulations of St_ßIB1 on the POPC and POPC:SM:CHOL bilayers (Fig. 3 and S3) the enzyme binds rapidly to the POPC:POPE bilayer (Fig. 5A). The density plots (Fig. 5B) show an anchoring slightly deeper than for the $\alpha$-clade enzymes in the PCcontaining bilayers and the orientation with respect to the bilayer surface is also slightly different with the flexible loop $\beta 6 \alpha 6$ being involved in interactions with the lipids (Fig. 5C \& D). This is reflected by the number of hydrophobic contacts and hydrogen bonds (Table 2). As a result of a deeper anchoring at the interface we observe more hydrophobic contacts with the lipid tails than between the $\alpha$ clade enzymes and PC bilayers. Amino acids of the highly conserved region of the flexible loop (I194, T195, L198, P199) all have on average more than 1.0 hydrophobic contacts per frame with the lipid tails in both replicas. The phenylalanine at position 54, which corresponds to a glycine in the two $\alpha$ clade enzymes, has a high count of hydrophobic contacts too.

We observe a dense hydrogen bond network between the lipids and several loops of St_ßIB1 (Table 2). In addition to the catalytic $(\beta 2 \alpha 2)$ and flexible $(\beta 6 \alpha 6)$ loops, the $\beta 7 \alpha 7$ loop and the amino acids of the $\alpha 8$ helix engage in long-lasting hydrogen bonds with lipid headgroups (occupancies between 50\% and 100\%). Loop $\beta 5 \alpha 5$ and helix $\alpha 7$ engage only in weak hydrogen bonds. Most of the hydrogen bonds involve the lipid phosphate group. However, six residues establish hydrogen bonds specifically with ammonium groups of the 
ethanolamine headgroups: N169, D201, D202, D229, E235 and E259 (Table 2). Among those D201 and D202 establish long-lasting hydrogen bonds with POPE ammonium groups (Fig. 6). The hydrogen bonds between other amino acids - N169, D229, E235, E259 - and POPE headgroups have a relatively lower occupancy ( $<40 \%$ of the simulation time). Interestingly in the two $\alpha$ clade enzymes, amino acids at position 202 are not acids but

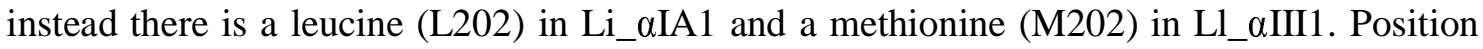

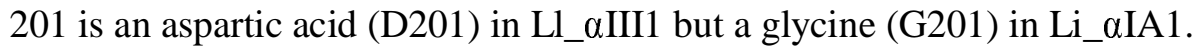

\section{FIGURE 6 HERE}

\section{(3) Engineering the aromatic cage in St_ßIB1 recovers binding to pure POPC bilayer}

\section{FIGURE 7 AND TABLE 3 HERE}

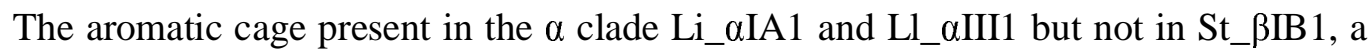
$\beta$ clade enzyme, is likely to be important for the recognition of choline groups of PC and SM lipids. We engineered such an aromatic box on St_ßIB1 by substituting R44 and S60 by tyrosines and simulated the resulting structure on a POPC bilayer. We checked that the mutations did not alter the protein structure. During the simulations we observed no major structural differences compared to the X-ray structure of the wild-type (Fig. S7). The backbone RMSD between the starting structure and the R44Y/S60Y double mutant oscillates between $1.0 \AA$ and $1.2 \AA$ during the production run, and the RMSD between WT St_ßIB1 Xray structure and R44Y/S60Y is equal to $1 \AA$ at the end of the simulation. Unlike what we

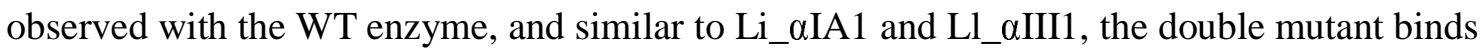
quickly to the POPC bilayer (Fig. 8). The interaction with lipids happens mostly at the 
catalytic loop (Fig. 7B-C). The aromatic cage interacts with choline headgroups and unlike for the wild type enzyme, few hydrogen bonds and hydrophobic contacts are established with the interfacial lipid groups (Table 3). This is in line with the shallow insertion of the enzyme. Overall, the interactions follow the same pattern as that obtained for Li_ $\alpha$ IA1 with the POPC bilayer.

\section{(4) Liposome Binding Assay}

\section{FIGURE 8 HERE}

Binding assays with mixed sphingomyelin:cholesterol (SM:CHOL) liposomes (Figure 8) provide some experimental support for the aromatic cage effect found in the simulations. La_ $\alpha$ IB2bi, an $\alpha$ clade protein possessing an aromatic cage and known to prefer substrates with choline head groups (14), is bound completely by 1:1 SM:CHOL liposomes. The $\beta$ clade enzyme St_ß1B1, which lacks an aromatic cage, is bound poorly, with an average of only 15 $\pm 4 \%$ of the protein sample pulled down by the liposomes relative to a negative control. The aromatic cage variant R44Y/S60Y St_ß1B1 showed higher but still incomplete binding, with $28 \pm 4 \%$ pulled down.

\section{(5) The aromatic cage is mostly conserved in $\alpha$ clade SicTox PLDs}

\section{FIGURE 9 HERE}

A multiple sequence alignment (MSA) of 25 PLDs from Lajoie et al. 2015 (14) and 7 additional samples that represent known diversity (list provided in methods section) shows that the sequences are fairly similar with the lowest sequence identity being around $37 \%$

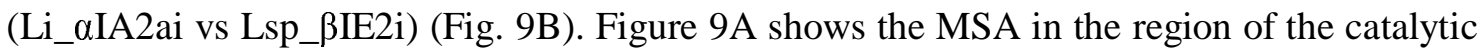
loop (from position 31 to 62, Fig. S8) and of the flexible loop (from position 192 to 204, Fig. 
S8). The full MSA is given in supporting information (Fig. S8).

The aromatic cage is mostly conserved in the $\alpha$ clade but the complete cage is mostly absent from the $\beta$ clade enzymes. Y44 and Y46 are strictly conserved in the $\alpha$ clade; position 60 can be either a tyrosine or a tryptophan except for Lg_alphaIC1 where it is a serine. The cage is not present in Lru_ $\alpha \mathrm{IC} 2$. In the $\beta$ clade, only Y46 is mostly conserved (except in Li_ßID1 and La_ßID1) but position 44 is a charged amino acid (E, R or K) and position 60 is a polar amino acid (S, T or Q). Position 62 is an aromatic (Y) in about half of the $\alpha$ clade PLDs of our dataset, forming an aromatic cage of four residues. In the $\beta$ clade sequences, this position is not occupied by aromatic residues. Only two representants of the $\beta$ clade exhibit the aromatic cage (Lsp_ßIII1 and Sd_ßIF).

In the flexible loop, only two $\beta$ clade enzymes present an aspartate at position 201 (G, I or $\mathrm{V}$ for the other $\beta$ clade PLDs and G or D for the $\alpha$ clade PLDs). D202 is conserved among the $\beta$ clade PLDs in our dataset (only two $\beta$ clade PLDs present a methionine at this position). Interestingly, this position is occupied by a hydrophobic residue in the $\alpha$ clade PLDs (M or L). D201 and D202 are located in the $\beta 6 \alpha 6$ loop (flexible loop). The C-terminus side of this loop is richer in aspartate in $\beta$ clade PLDs than in the $\alpha$ clade PLDs towards the end of this loop (Fig. 9).

\section{DISCUSSION AND CONCLUSIONS}

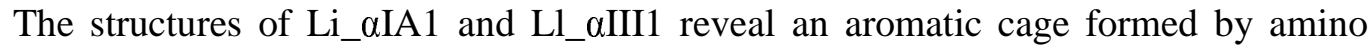
acids at positions 44,46 and 60 , but this cage is not present in the Sicarius PLD (St_ßIB1) structure, leading to the hypothesis that this cage is part of the mechanism by which the $\alpha$ clade enzymes achieve high affinity for choline-containing lipid headgroups. We discuss 
below how our results support this hypothesis.

MD simulations showed that Li_aIA1 and Ll_aIII1 bind to choline-containing bilayers with the same binding orientation whereas St_ßIB1 remained in solvent. The i-face of the two $\alpha$ clade enzymes on choline-containing bilayers involves the catalytic loop and cation- $\pi$ interactions between the aromatic residues forming the cage and choline headgroup. The aromatic residues located at the i-face are engaged in strong cation- $\pi$ interactions with choline headgroups. The liposome binding assays show that La_aIB2bi, an $\alpha$ clade enzyme with the aromatic cage, shows strong binding to SM:CHOL (1:1) liposomes whereas St_ $\beta$ IB1 binds poorly to those.

The structure of the aromatic cage resembles aromatic cages found in other proteins around choline groups $(27,30,59-61)$. Such cages consist of 2 to 4 aromatic side chains, either only tyrosines as in $\mathrm{Li} \alpha \mathrm{IA} 1$, or a combination of tyrosines and tryptophans as we observe L1_aIII1. Roughly half the sequences in our datasets have a cage consisting of three aromatics and half have a cage of four. The aromatic amino acids arrange as a box forming a hydrophobic binding site for the methylated ammonium which positive charge is also stabilized by the $\pi$-system of the aromatics groups. The shortest distances between carbons of the aromatic ring and methyl groups are within $4 \AA$. Examples of cages around cholinecontaining lipids include the structure of the human phosphatidylcholine transfer protein (PDB IDs 1LN1 and 1LN3) complexed with 1,2-dilinoleoyl-sn-glycerol-3-phosphorylcholine (DLPC,18:2(9,12)-18:2(9,12)) or 1-palmitoyl,2-linoleoyl-sn-glycerol-3-phosphorylcholine (PLPC, 16:0-18:2(9,12)) bound to a cage consisting of three tyrosines and a tryptophan. In the engineered N254Y/H258Y SaPI-PLC two tyrosines and one tryptophan bind a choline ion (PDB ID 4I90) or the choline group of diC4PC (PDB ID 4I9J)(27).

Inspired by the work of Cheng et al, we engineered an aromatic cage in the $\beta$ clade 
enzyme in silico (27). Starting from Staphylococcus aureus PI-PLC (SaPI-PLC) which does not bind to PC-rich vesicles, Cheng et al increased its affinity for PC-rich liposomes by engineering an aromatic cage inspired by the sequence and structure of the PC-specific homologue BtPI-PLC. X-ray structures of the modified SaPI-PLC enzyme soaked with phosphocholine or $\mathrm{DiC}_{7} \mathrm{PC}$ (1,2-Diheptanoyl-sn-Glycero-3-Phosphocholine) revealed that the choline groups were positioned in the aromatic cage. Our simulations of the engineered St_ßIB1 (R44Y/S60Y St_ßIB1) lead to a stable interaction with a POPC bilayer, unlike the results we obtained with the wild-type. Just as we observed for the $\alpha$ clade enzymes, a choline headgroup was observed residing in the aromatic cage during most of the simulation time. The liposome binding assay of R44Y/S60Y St_ßIB1on SM:CHOL (1:1) liposomes shows significantly increased binding compared to WT St_ßIB1. This suggests that the aromatic cage is one important factor for the binding of membranes rich in choline head groups; on the other hand, the lack of complete pulldown suggests that other factors must also contribute to the weak binding of St_ß1B1 to choline-rich membranes, relative to $\alpha$ clade enzymes such as La_ $\alpha$ IB2bi.

An alignment of the sequences of 25 representative PLDs from different subgroups of the two clades shows that the aromatic cage is highly conserved in the PLDs from the $\alpha$ clade and not present in the well characterized PLDs from the $\beta$ clade. Overall, the conservation of a cage in most $\alpha$-clade enzymes, and its positive role in choline recognition based on simulations and in silico and in vitro mutagenesis data, support a significant role for the cage in targeting $\alpha$-clade toxins to choline-rich membranes. Other things being equal, this targeting should increase access of the $\alpha$-clade enzymes to the choline-containing substrates SM and lysophosphatidylcholine (LPC), toward which they have generally high activity. This likely contributes to the efficacy of $\alpha$-clade toxins in triggering loxoscelism in humans, but presumably also plays an adaptive role in prey capture, the principal biological function of 
Loxosceles venom (20). Meanwhile, St_ßIB1, a $\beta$ clade paralog that strongly prefers substrates with ethanolamine head groups, lacks the cage, as do most other $\beta$ clade enzymes. In both simulation and experiment, its binding to choline-rich membranes is increased by introduction of the cage. One caveat is that La_ßID1, a second $\beta$ clade enzyme that also lacks the cage, has fairly high activity against choline-containing substrates, though low preference relative to ethanolamine-containing substrates (14) suggesting that other determinants of head group preference must also contribute.

To better understand the specificity of St_ßIB1 for PE-rich membrane, we also ran simulations of WT St_ßIB1 in the presence of a POPC:POPE (50:50) bilayer. As opposed to the simulation on bilayers without POPE lipids, WT St_ßIB1 bound to the bilayer with an iface composed of the catalytic ( $\beta 2 \alpha 2)$ and flexible ( $\beta 6 \alpha 6)$ loops, as well as loop $\beta 7 \alpha 7$ and helix $\alpha 8$. WT St_ßIB1 is anchored slightly deeper in the bilayer than the $\alpha$-clade enzymes and in lieu of cation-pi interactions, it engages in a dense hydrogen bond network with the lipid phosphates and headgroups. Interestingly two aspartates located on the flexible loop establish hydrogen bonds with the ethanolamine headgroup. Early assessments of sequence diversity in the MSA indicate that the-terminus region of the catalytic loop is richer in aspartate in the $\beta$ clade than in the $\alpha$ clade.

Beyond suggesting the molecular basis for the specificity of $\alpha$-clade PLDs for choline-rich membranes, this work is relevant for the design of drugs targeting PLDs from SicTox spiders. We suggest that the aromatic cage might be worth targeting to weaken protein-membrane interactions of the $\alpha$ clade enzymes. Last but not least the role of the evolutionary conserved aromatic cage in SicTox PLD enzymes, together with already existing evidence of interfacial choline-aromatics cation- $\pi$ interactions in other protein families $(24$, $25,60,62)$, further confirms that such interactions could be a general mechanism for specific 
recognition of the surface of eukaryotic cells or other choline-rich biological membranes.

\section{Acknowledgments}

This work was supported by the Research Council of Norway through grants \#251247 and \#288008 to NR, and by the National Science Foundation through grant \#1808716 to MC.

\section{REFERENCES}

1. Dantas, A.E., A.O. Carmo, C.C.R. Horta, H.G. Leal, B.B.R. Oliveira-Mendes, A.P.V. Martins, C. Chávez-Olórtegui, and E. Kalapothakis. 2016. Description of Loxtox protein family and identification of a new group of Phospholipases D from Loxosceles similis venom gland. Toxicon. 120: 97-106.

2. Da Silva, P.H., R.B. Da Silveira, M. Helena Appel, O.C. Mangili, W. Gremski, and S.S. Veiga. 2004. Brown spiders and loxoscelism. Toxicon. 44: 693-709.

3. Wille, A.C.M., D. Chaves-Moreira, D. Trevisan-Silva, M.G. Magnoni, M. BoiaFerreira, L.H. Gremski, W. Gremski, O.M. Chaim, A. Senff-Ribeiro, and S.S. Veiga. 2013. Modulation of membrane phospholipids, the cytosolic calcium influx and cell proliferation following treatment of B16-F10 cells with recombinant phospholipase-D from Loxosceles intermedia (brown spider) venom. Toxicon. 67: 17-30.

4. Magalhaes, I.L., A.D. Brescovit, and A. Santos. 2017. Phylogeny of Sicariidae spiders (Araneae: Haplogynae), with a monograph on Neotropical Sicarius. Zool. J. Linn. Soc. 179: 767-864.

5. Binford, G.J., M.R. Bodner, M.H.J. Cordes, K.L. Baldwin, M.R. Rynerson, S.N. Burns, and P.A. Zobel-Thropp. 2009. Molecular evolution, functional variation, and proposed nomenclature of the gene family that includes sphingomyelinase D in sicariid spider venoms. Mol. Biol. Evol. 26: 547-566.

6. Tambourgi, D. V., F.C. Magnoli, C.W. van den Berg, B.P. Morgan, P.S. de Araujo, E.W. Alves, and W.D. Da Silva. 1998. Sphingomyelinases in the Venom of the Spider Loxosceles intermediaAre Responsible for both Dermonecrosis and ComplementDependent Hemolysis. Biochem. Biophys. Res. Commun. 251: 366-373.

7. Chaves-Moreira, D., A. Senff-Ribeiro, A.C.M. Wille, L.H. Gremski, O.M. Chaim, and S.S. Veiga. 2017. Highlights in the knowledge of brown spider toxins. J. Venom. Anim. Toxins Incl. Trop. Dis. 23: 1-12.

8. Chaves-Moreira, D., F.R. de Moraes, Í.P. Caruso, O.M. Chaim, A. Senff-Ribeiro, A. Ullah, L.S. da Silva, J. Chahine, R.K. Arni, and S.S. Veiga. 2017. Potential Implications for Designing Drugs Against the Brown Spider Venom Phospholipase-D. J. Cell. Biochem. 118: 726-738.

9. P.Gopalakrishnakone, G.C., and E.-G. Maria Elena de Lima. Spider venoms. Springer.

10. Lajoie, D.M., P.A. Zobel-Thropp, V.K. Kumirov, V. Bandarian, G.J. Binford, and M.H.J. Cordes. 2013. Phospholipase D Toxins of Brown Spider Venom Convert Lysophosphatidylcholine and Sphingomyelin to Cyclic Phosphates. PLoS One. 8: 1-7.

11. Murakami, M.T., M. Freitas Fernandes-Pedrosa, S.A. de Andrade, A. Gabdoulkhakov, C. Betzel, D. V. Tambourgi, and R.K. Arni. 2006. Structural insights into the catalytic mechanism of sphingomyelinases $\mathrm{D}$ and evolutionary relationship to 
glycerophosphodiester phosphodiesterases. Biochem. Biophys. Res. Commun. 342: 323-329.

12. de Giuseppe, P.O., A. Ullah, D.T. Silva, L.H. Gremski, A.C.M. Wille, D. Chaves Moreira, A.S. Ribeiro, O.M. Chaim, M.T. Murakami, S.S. Veiga, and R.K. Arni. 2011. Structure of a novel class II phospholipase D: Catalytic cleft is modified by a disulphide bridge. Biochem. Biophys. Res. Commun. 409: 622-627.

13. Binford, G.J., M.R. Bodner, M.H.J. Cordes, K.L. Baldwin, M.R. Rynerson, S.N. Burns, and P.A. Zobel-Thropp. 2009. Molecular evolution, functional variation, and proposed nomenclature of the gene family that includes sphingomyelinase D in sicariid spider venoms. Mol. Biol. Evol. 26: 547-566.

14. Lajoie, D.M., S.A. Roberts, P.A. Zobel-Thropp, J.L. Delahaye, V. Bandarian, G.J. Binford, and M.H.J. Cordes. 2015. Variable Substrate Preference among Phospholipase D Toxins from Sicariid Spiders. J. Biol. Chem. 290: 10994-11007.

15. de Santi Ferrara, G.I., M. de F. Fernandes-Pedrosa, I. de L.M. Junqueira-de-Azevedo, R.M. Gonçalves-de-Andrade, F.C.V. Portaro, D. Manzoni-de-Almeida, M.T. Murakami, R.K. Arni, C.W. van den Berg, P.L. Ho, and D. V. Tambourgi. 2009. SMase II, a new sphingomyelinase D from Loxosceles laeta venom gland: Molecular cloning, expression, function and structural analysis. Toxicon. 53: 743-753.

16. Ramos-Cerrillo, B., A. Olvera, G. V. Odell, F. Zamudio, J. Paniagua-Solís, A. Alagón, and R.P. Stock. 2004. Genetic and enzymatic characterization of sphingomyelinase D isoforms from the North American fiddleback spiders Loxosceles boneti and Loxosceles reclusa. Toxicon. 44: 507-514.

17. da Silveira, R.B., R.B. Pigozzo, O.M. Chaim, M.H. Appel, D.T. Silva, J.L. Dreyfuss, L. Toma, C.P. Dietrich, H.B. Nader, S.S. Veiga, and W. Gremski. 2007. Two novel dermonecrotic toxins LiRecDT4 and LiRecDT5 from Brown spider (Loxosceles intermedia) venom: From cloning to functional characterization. Biochimie. 89: 289300.

18. Olvera, A., B. Ramos-Cerrillo, J. Estévez, H. Clement, A. de Roodt, J. Paniagua-Solís, H. Vázquez, A. Zavaleta, M. Salas Arruz, R.P. Stock, and A. Alagón. 2006. North and South American Loxosceles spiders: Development of a polyvalent antivenom with recombinant sphingomyelinases $\mathrm{D}$ as antigens. Toxicon. 48: 64-74.

19. Bertoni da Silveira, R., R.B. Pigozzo, O.M. Chaim, M.H. Appel, J.L. Dreyfuss, L. Toma, O.C. Mangili, W. Gremski, C.P. Dietrich, H.B. Nader, and S.S. Veiga. 2006. Molecular cloning and functional characterization of two isoforms of dermonecrotic toxin from Loxosceles intermedia (Brown spider) venom gland. Biochimie. 88: 12411253.

20. Zobel-Thropp, P.A., A.E. Kerins, and G.J. Binford. 2012. Sphingomyelinase D in Sicariid Spider Venom is a Potent Insecticidal Toxin. Toxicon. 60: 265-271.

21. Khan, H.M., T. He, E. Fuglebakk, C. Grauffel, B. Yang, M.F. Roberts, A. Gershenson, and N. Reuter. 2016. A Role for Weak Electrostatic Interactions in Peripheral Membrane Protein Binding. Biophys. J. 110: 1367-1378.

22. Schillinger, A.S., C. Grauffel, H.M. Khan, Ø. Halskau, and N. Reuter. 2014. Two homologous neutrophil serine proteases bind to POPC vesicles with different affinities: When aromatic amino acids matter. Biochim. Biophys. Acta - Biomembr. 1838: 3191-3202.

23. Aksnes, H., M. Goris, Ø. Strømland, A. Drazic, Q. Waheed, N. Reuter, and T. Arnesen. 2017. Molecular determinants of the N-Terminal acetyltransferase Naa60 anchoring to the Golgi membrane. J. Biol. Chem. 292: 6821-6837.

24. Weber, D.K., S. Yao, N. Rojko, G. Anderluh, T.P. Lybrand, M.T. Downton, J. Wagner, and F. Separovic. 2015. Characterization of the lipid-binding site of 
equinatoxin II by NMR and molecular dynamics simulation. Biophys. J. 108: 19871996.

25. Grauffel, C., B. Yang, T. He, M.F. Roberts, A. Gershenson, and N. Reuter. 2013. Cation- $\pi$ interactions as lipid-specific anchors for phosphatidylinositol-specific phospholipase C. J. Am. Chem. Soc. 135: 5740-5750.

26. Roberts, M.F., H.M. Khan, R. Goldstein, N. Reuter, and A. Gershenson. 2018. Search and Subvert: Minimalist Bacterial Phosphatidylinositol-Specific Phospholipase C Enzymes. Chem. Rev. 118: 8435-8473.

27. Cheng, J., R. Goldstein, A. Gershenson, B. Stec, and M.F. Roberts. 2013. The cation- $\pi$ box is a specific phosphatidylcholine membrane targeting motif. J. Biol. Chem. 288: $14863-14873$.

28. Waheed, Q., H.M. Khan, T. He, M. Roberts, A. Gershenson, and N. Reuter. 2019. Interfacial Aromatics Mediating Cation- $\pi$ Interactions with Choline-Containing Lipids Can Contribute as Much to Peripheral Protein Affinity for Membranes as Aromatics Inserted below the Phosphates. J. Phys. Chem. Lett. 10: 3972-3977.

29. Celie, P.H.., S.E. van Rossum-Fikkert, W.J. van Dijk, K. Brejc, A.B. Smit, and T.K. Sixma. 2004. Nicotine and Carbamylcholine Binding to Nicotinic Acetylcholine Receptors as Studied in AChBP Crystal Structures. Neuron. 41: 907-914.

30. Roderick, S.L., W.W. Chan, D.S. Agate, L.R. Olsen, M.W. Vetting, K.R. Rajashankar, and D.E. Cohen. 2002. Structure of human phosphatidylcholine transfer protein in complex with its ligand. Nat. Struct. Biol. 9: 507-511.

31. Beene, D.L., G.S. Brandt, W. Zhong, N.M. Zacharias, H.A. Lester, and D.A. Dougherty. 2002. Cation- $\pi$ interactions in ligand recognition by serotonergic (5HT3A) and nicotinic acetylcholine receptors: The anomalous binding properties of nicotine. Biochemistry. .

32. Berman, H.M., T. Battistuz, T.N. Bhat, W.F. Bluhm, P.E. Bourne, K. Burkhardt, Z. Feng, G.L. Gilliland, L. Iype, S. Jain, P. Fagan, J. Marvin, D. Padilla, V.

Ravichandran, B. Schneider, N. Thanki, H. Weissig, J.D. Westbrook, and C. Zardecki. 2002. The protein data bank. Acta Crystallogr. Sect. D Biol. Crystallogr. 58: 899-907.

33. Arun S., K., W. James C., S. Peter J., and A.M. Lesk. 2006. MUSTANG: A multiple structural alignment algorithm. Sci. Technol. 64: 559-574.

34. Madeira, F., Y.M. Park, J. Lee, N. Buso, T. Gur, N. Madhusoodanan, P. Basutkar, A.R.N. Tivey, S.C. Potter, R.D. Finn, and R. Lopez. 2019. The EMBL-EBI search and sequence analysis tools APIs in 2019. Nucleic Acids Res. 47: W636-W641.

35. Sunhwan Jo, Taehoon Kim, Vidyashankara G. Iyer, W.I. 2008. Software News and Updates CHARMM-GUI: A Web-Based Graphical User Interface for CHARMM. J. Comput. Chem. 29: 859-1865.

36. Wu, E.L., X. Cheng, S. Jo, H. Rui, K.C. Song, E.M. Dávila-Contreras, Y. Qi, J. Lee, V. Monje-Galvan, R.M. Venable, J.B. Klauda, and W. Im. 2014. CHARMM-GUI membrane builder toward realistic biological membrane simulations. J. Comput. Chem. 35: 1997-2004.

37. Jo, S., J.B. Lim, J.B. Klauda, and W. Im. 2009. CHARMM-GUI membrane builder for mixed bilayers and its application to yeast membranes. Biophys. J. 97: 50-58.

38. Lee, J., D.S. Patel, J. Ståhle, S.J. Park, N.R. Kern, S. Kim, J. Lee, X. Cheng, M.A. Valvano, O. Holst, Y.A. Knirel, Y. Qi, S. Jo, J.B. Klauda, G. Widmalm, and W. Im. 2019. CHARMM-GUI Membrane Builder for Complex Biological Membrane Simulations with Glycolipids and Lipoglycans. J. Chem. Theory Comput. 15: 775786.

39. Klauda, J.B., R.M. Venable, J.A. Freites, J.W. O’Connor, D.J. Tobias, C. MondragonRamirez, I. Vorobyov, A.D. MacKerell, and R.W. Pastor. 2010. Update of the 
CHARMM All-Atom Additive Force Field for Lipids: Validation on Six Lipid Types. J. Phys. Chem. B. 114: 7830-7843.

40. $\quad$ Best, R.B., X. Zhu, J. Shim, P.E.M. Lopes, J. Mittal, M. Feig, and A.D. MacKerell. 2012. Optimization of the additive CHARMM all-atom protein force field targeting improved sampling of the backbone $\varphi, \psi$ and side-chain $\chi 1$ and $\chi 2$ Dihedral Angles. J. Chem. Theory Comput. 8: 3257-3273.

41. MacKerell, A.D., D. Bashford, M. Bellott, R.L. Dunbrack, J.D. Evanseck, M.J. Field, S. Fischer, J. Gao, H. Guo, S. Ha, D. Joseph-McCarthy, L. Kuchnir, K. Kuczera, F.T.K. Lau, C. Mattos, S. Michnick, T. Ngo, D.T. Nguyen, B. Prodhom, W.E. Reiher, B. Roux, M. Schlenkrich, J.C. Smith, R. Stote, J. Straub, M. Watanabe, J.

Wiórkiewicz-Kuczera, D. Yin, and M. Karplus. 1998. All-atom empirical potential for molecular modeling and dynamics studies of proteins. J. Phys. Chem. B. 102: 35863616.

42. Kučerka, N., S. Tristram-Nagle, and J.F. Nagle. 2006. Structure of fully hydrated fluid phase lipid bilayers with monounsaturated chains. J. Membr. Biol. 208: 193-202.

43. Saeedimasine, M., A. Montanino, S. Kleiven, and A. Villa. 2019. Role of lipid composition on the structural and mechanical features of axonal membranes: a molecular simulation study. Sci. Rep. 9: 8000.

44. Pitman, M.C., F. Suits, A.D. MacKerell, and S.E. Feller. 2004. Molecular-Level Organization of Saturated and Polyunsaturated Fatty Acids in a Phosphatidylcholine Bilayer Containing Cholesterol †. Biochemistry. 43: 15318-15328.

45. Pettersen, E.F., T.D. Goddard, C.C. Huang, G.S. Couch, D.M. Greenblatt, E.C. Meng, and T.E. Ferrin. 2004. UCSF Chimera - A visualization system for exploratory research and analysis. J. Comput. Chem. 25: 1605-1612.

46. Lomize, M.A., I.D. Pogozheva, H. Joo, H.I. Mosberg, and A.L. Lomize. 2012. OPM database and PPM web server: Resources for positioning of proteins in membranes. Nucleic Acids Res. 40: 370-376.

47. Humphrey, W., A. Dalke, and K. Schulten. 1996. VMD: Visual Molecular Dynamics. J. Mol. Graph. 14: 33-38.

48. Phillips, J.C., R. Braun, W. Wang, J. Gumbart, E. Tajkhorshid, E. Villa, C. Chipot, R.D. Skeel, L. Kalé, and K. Schulten. 2005. Scalable molecular dynamics with NAMD. J. Comput. Chem. 26: 1781-1802.

49. Khan, H.M., C. Grauffel, R. Broer, A.D. MacKerell, R.W.A. Havenith, and N. Reuter. 2016. Improving the Force Field Description of Tyrosine-Choline Cation- $\pi$ Interactions: QM Investigation of Phenol-N(Me)4+ Interactions. J. Chem. Theory Comput. 12: 5585-5595.

50. Khan, H.M., A.D. MacKerell, and N. Reuter. 2019. Cation- $\pi$ Interactions between Methylated Ammonium Groups and Tryptophan in the CHARMM36 Additive Force Field. J. Chem. Theory Comput. 15: 7-12.

51. Andersen, H.C. 1983. Rattle: A "velocity" version of the shake algorithm for molecular dynamics calculations. J. Comput. Phys. 52: 24-34.

52. Essmann, U., L. Perera, M.L. Berkowitz, T. Darden, H. Lee, and L.G. Pedersen. 1995. A smooth particle mesh Ewald method. J. Chem. Phys. 103: 8577-8593.

53. Brooks, B.R., C.. Brooks, A.D. Mackerell, L. Nilsson, R.J. Petrella, B. Roux, Y. Won, G. Archontis, C. Bartels, S. Boresch, A. Caflisch, L. Caves, Q. Cui, A.R. Dinner, M. Feig, S. Fischer, J. Gao, M. Hodoscek, W. Im, K. Kuczera, T. Lazaridis, J. Ma, V. Ovchinnikov, E. Paci, R.W. Pastor, C.B. Post, J.Z. Pu, M. Schaefer, B. Tidor, R.M. Venable, H.L. Woodcock, X. Wu, W. Yang, Y. D.M, and M. Karplus. 2009. CHARMM: Molecular dynamics simulation package. J. Comput. Chem. 30: 15451614. 
54. Michaud-Agrawal, N., T.B.W. E. J. Denning, and O. Beckstein. 2011. MDAnalysis: A Toolkit for the Analysis of Molecular Dynamics Simulations. ... Comput. Chem. 32: 2319-2327.

55. Gowers, R., M. Linke, J. Barnoud, T. Reddy, M. Melo, S. Seyler, J. Domański, D. Dotson, S. Buchoux, I. Kenney, and O. Beckstein. 2016. MDAnalysis: A Python Package for the Rapid Analysis of Molecular Dynamics Simulations. Proc. 15th Python Sci. Conf. : 98-105.

56. Zobel-Thropp, P.A., A.E. Kerins, and G.J. Binford. 2012. Sphingomyelinase D in sicariid spider venom is a potent insecticidal toxin. Toxicon. 60: 265-271.

57. Lajoie, D.M., and M.H.J. Cordes. 2015. Spider, bacterial and fungal phospholipase D toxins make cyclic phosphate products. Toxicon. 108: 176-180.

58. Schneider, C.A., W.S. Rasband, and K.W. Eliceiri. 2012. NIH Image to ImageJ: 25 years of image analysis. Nat. Methods. 9: 671-5.

59. Dougherty, D.A., and D.A. Stauffer. 1990. Acetylcholine binding by a synthetic receptor: Implications for biological recognition. Science (80-. ). .

60. Goh, B.C., H. Wu, M.J. Rynkiewicz, K. Schulten, B.A. Seaton, and F.X. McCormack. 2016. Elucidation of Lipid Binding Sites on Lung Surfactant Protein A Using X-ray Crystallography, Mutagenesis, and Molecular Dynamics Simulations. Biochemistry. 55: 3692-3701.

61. Pittelkow, M., B. Tschapek, S.H.J. Smits, L. Schmitt, and E. Bremer. 2011. The Crystal Structure of the Substrate-Binding Protein OpuBC from Bacillus subtilis in Complex with Choline. J. Mol. Biol. 411: 53-67.

62. Hirano, Y., Y.-G. Gao, D.J. Stephenson, N.T. Vu, L. Malinina, D.K. Simanshu, C.E. Chalfant, D.J. Patel, and R.E. Brown. 2019. Structural basis of phosphatidylcholine recognition by the $\mathrm{C} 2$-domain of cytosolic phospholipase A2 $\alpha$. Elife. 8 . 


\section{Tables}

Table 1. Inventory of interactions between Li_aIA1 (3RLH) or LI_aIII1 (1XX1) and two types of bilayers: POPC and POPC:PSM:CHOL. Hydrophobic contacts are given as average number of contacts per frame. Hydrogen bonds and cation- $\pi$ occupancies are shown in percentages. Residues in bold indicate hydrogen bonds involving the amino acid backbone.

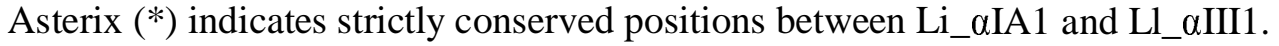

\begin{tabular}{|c|c|c|c|c|c|}
\hline \multirow{3}{*}{ SSE } & \multirow{3}{*}{ residue } & \multicolumn{2}{|c|}{ Li_aIA1 } & \multicolumn{2}{|c|}{ Ll_aIII1 } \\
\hline & & Bilayer A & Bilayer B & Bilayer A & Bilayer B \\
\hline & & R1 & R1 & R1 & R1 \\
\hline
\end{tabular}

\section{Hydrogen bonds (\%)}

\begin{tabular}{lccccccccc}
\hline$\beta 2 \alpha 2$ & K38 & - & - & - & - & 33.56 & 36.54 & 40.0 & 36.70 \\
& Y44* & 15.53 & 20.76 & 24.89 & 21.98 & - & 15 & 24.33 & 16.87 \\
& Y46* & 67.96 & 63.45 & 50.34 & 50.34 & - & - & - & - \\
& I49 & 23.21 & 21.89 & 20.12 & 21.67 & - & - & - & - \\
& C51* & 45.93 & 40.78 & 38.56 & 35.56 & - & - & - & - \\
& G54 & 19.98 & 26.37 & 35.90 & 35.77 & - & - & - & - \\
& K58 & 72.49 & 79.90 & 83.21 & 77.45 & - & - & - & - \\
& R59 & - & - & - & - & 91.45 & 89.45 & 100 & 92.45 \\
& W60 & - & - & - & - & 15.45 & 11 & 15 & 20.56 \\
& Y62 & - & - & - & - & 50.34 & 34.78 & 45.0 & 46.65 \\
$\beta 3 \alpha 3$ & Y98 & 36.96 & 46.89 & 35.90 & 32.87 & - & - & - & - \\
\hline
\end{tabular}

Hydrophobic contacts (avg. contact per frame)

\begin{tabular}{cccccccccc}
\hline$\beta 2 \alpha 2$ & I49 & 4.0 & 4.2 & 5.0 & 4.0 & - & - & - & - \\
& P50* & 3.0 & 3.4 & 4.3 & 3.2 & - & - & - & - \\
& I58 & - & - & - & - & 1.0 & 0.6 & 0.6 & 0.7 \\
\hline
\end{tabular}

cation- $\pi$ interactions $(\%)$

\begin{tabular}{rccccccccc}
\hline$\beta 2 \alpha 2$ & Y44* & 35.6 & 28.90 & 30.54 & 34.78 & 28.8 & 27.54 & 34.89 & 29.63 \\
& Y46* & 40.4 & 46.00 & 43.76 & 44.34 & 73.9 & 67.90 & 84.23 & 86.33 \\
& Y60/W60 & 44.7 & 44.78 & 40.6 & 33.23 & 84.4 & 80.27 & 83.12 & 89.00 \\
& Y62 & - & - & - & - & 41.2 & 45.89 & 39.45 & 41.67 \\
$\beta 3 \alpha 3$ & Y98 & 50.5 & 60.63 & 46.65 & 47.86 & - & - & - & - \\
\hline
\end{tabular}


Table 2. Inventory of interactions between WT St_ßIB1 and a POPC:POPE bilayer. Hydrophobic contacts are given as average number of contacts per frame. Hydrogen bonds and cation- $\pi$ occupancies are shown in percentages. Residues in bold indicate hydrogen bonds involving the amino acid backbone. Underlined residue names indicate hydrogen bonds involving the ethanolamine headgroup.

\begin{tabular}{lccc}
\hline SSE & Residue & R1 & R2 \\
\hline \multirow{4}{*}{$\beta 2 \alpha 2$} & & \multicolumn{2}{c}{ Hydrogen bonds (\%) } \\
& & 99.83 & 57.94 \\
& C51 & 17.25 & 19.70 \\
$\beta 5 \alpha 5$ & C53 & 99.67 & 99.85 \\
$\beta 6 \alpha 6$ & S56 & 40.63 & 41.76 \\
& T58 & 32.17 & 22.10 \\
$\beta 7 \alpha 7$ & R59 & 29.85 & 12.94 \\
$\alpha 7$ & R200 & 12.44 & 49.26 \\
& D201 & 80.54 & 85.38 \\
& D202 & 58.87 & 65.67 \\
& $\underline{D} 229$ & 29.02 & 13.08 \\
& K230 & 68.16 & 60.58 \\
$\alpha 8$ & E231 & 33.51 & 36.32 \\
& S232 & 56.38 & 37.50 \\
& S233 & 40.46 & 40.00 \\
& E235 & 40.83 & 34.41 \\
& Y249 & 76.12 & 84.85 \\
& R252 & 85.41 & 81.47 \\
& E259 & 27.36 & 32.50 \\
& R260 & 30.51 & 36.18 \\
\hline
\end{tabular}

Hydrophobic contacts (avg. contact per frame)

\begin{tabular}{llll}
\hline$\beta 2 \alpha 2$ & P50 & 0.14 & 0.10 \\
& C51 & 1.23 & 0.78 \\
& C53 & 0.66 & 0.45 \\
& F54 & 1.45 & 1.30 \\
$\beta 6 \alpha 6$ & R55 & 1.03 & 0.78 \\
& I194 & 1.02 & 1.11 \\
& T195 & 1.13 & 1.22 \\
& C197 & 0.75 & 0.60 \\
$\alpha 7$ & L198 & 1.53 & 1.67 \\
& P199 & 1.62 & 1.53 \\
& K230 & 1.34 & 1.22 \\
\hline
\end{tabular}


Table 3. Inventory of interactions between R44Y/S60Y St_BIB1 and a POPC bilayer. Hydrophobic contacts are given as average number of contacts per frame. Hydrogen bonds and cation- $\pi$ occupancies are shown in percentages. Residues in bold indicate hydrogen bonds involving the amino acid backbone.

\begin{tabular}{cccc}
\hline SSE & Residues & R1 & R2 \\
\hline \multirow{4}{*}{ Hydrogen bonds (\%) } \\
\hline$\beta 2 \alpha 2$ & K38 & 39.085 & 68.49 \\
& Y44 & 10.54 & 20.78 \\
& Y46 & 60.00 & 54.34 \\
& C51 & 53.33 & 67.34 \\
S56 & 46.67 & 58.34 \\
& C57 & 20.60 & 10.32 \\
& Y60 & 20.00 & 35.81 \\
\hline
\end{tabular}

Hydrophobic contacts (avg. contact per frame)

\begin{tabular}{cccc}
\hline$\beta 2 \alpha 2$ & V49 & 3.0 & 4.3 \\
& P50 & 2.5 & 1.9 \\
\hline \multicolumn{4}{c}{ cation- $\pi$ interactions (\%) } \\
\hline \multirow{4}{*}{$\beta 2 \alpha 2$} & Y44 & 43.62 & 39.89 \\
& Y46 & 35.63 & 40.23 \\
& Y60 & 44.81 & 20.33 \\
\hline
\end{tabular}




\section{Figure captions}

Figure 1. Overview on the SicTox PLDs structure. A) Structures of phospholipades D used

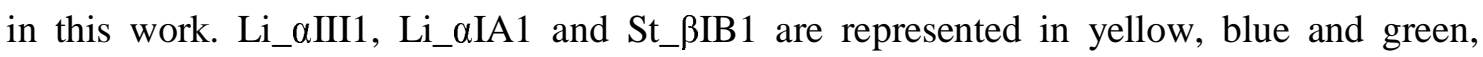
respectively. The side chains of amino acids critical in substrate binding and catalysis are shown in orange. The aromatic residues located at the putative i-face are indicated in purple. B) PLD topology: strands (blue) and helices (red) of canonical $(\alpha / \beta)_{8}$ barrel, other secondary structure elements (green), catalytic loop (cyan), flexible loop (green), disulphide bridges (purple). Residue numbering is based on the Li_aIA1 structure (PDB id: 3RLH). C) Structure and sequence similarity: pairwise RMSD (lower left triangle) and sequence similarity (upper right part).

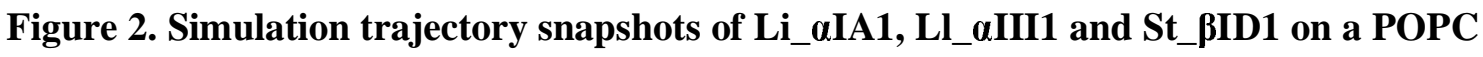
bilayer. Snapshots were taken at time $\mathrm{t}=0,80,160,270$ and $300 \mathrm{~ns}$ of the production run.

Figure 3. Li_aIA1 and LI_aIII1 bound to POPC and POPC:PSM:CHOL (70:20:10). A)

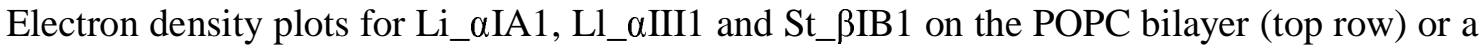
the POPC:PSM:CHOL bilayer (bottom row), replica 1 (solid lines) and replica 2 (dashed lines). B) Bound orientation of Li_aIA1 on a pure POPC bilayer. C) Binding orientation of

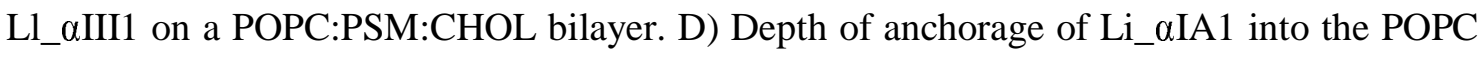
bilayer. The depth is calculated for each amino acid as the distance between its C-alpha and the phosphate plane in the last frame of simulation (relevant values are given in Tables S4 \& S5).

Figure 4. Phosphatidylcholine headgroups trapped in the aromatic cage of Li_aIA1 (blue) and Ll_aIII1 (yellow). Lipids carbon atoms are in purple. The snapshots are extracted from the last $150 \mathrm{~ns}$ of the simulation trajectories. 
Figure 5. Binding of St_ßIB1 to a POPC:POPE (50:50) bilayer. A) Simulation snapshots.

B) Electron density plot for two replica (solid lines and dashed lines). C) Bound form. D)

Depth of anchorage. The depth is calculated for each amino acid as the distance between its C-alpha and the phosphate plane in the last frame of simulation (relevant values are given in Table S6).

Figure 6. Hydrogen bonds (yellow) between residues D201 and D202 of St_ßID1 and phosphatidylethanolamine headgroups: POPE lipid (carbon atoms in purple), protein (green). The two snapshots are taken during the last $50 \mathrm{~ns}$ of simulation.

Figure 7. Binding of R44Y/S60Y St_ßIB1 to a POPC Bilayer. A) Simulation snapshots. B) Electron density plot for R44Y/S60Y St_BIB1 and the POPC bilayer for two replica (solid lines and dashed lines). C) Bound protein orientation. D) Depth of anchorage. The depth is calculated for each amino acid as the distance between its $\mathrm{C}$-alpha and the phosphate plane in the last frame of simulation (relevant values are given in Table S4).

Figure 8. Liposome binding assay for WT La_aIB2bi, WT St_ßIB1 and R44Y/S60Y St_ßIB1 on SM:CHOL (1:1) liposomes. $P$ and $S$ refer to pellet and supernatant, respectively. The "-" symbol indicates the control lanes (without liposomes) and the "+" symbol the lanes with the protein and the liposomes. Across three experiments the mutant R44Y/S60Y St_BIB1 showed 10-17\% higher levels of pulldown than the WT, giving a mean increase of $13 \pm 2 \%$.

Figure 9. Amino acid sequences similarities in the catalytic and flexible loops. A) Multiple sequence alignment of the catalytic and the flexible loop. The full sequence alignment is given as supporting information (Fig S8). B) Sequence similarity matrix. 


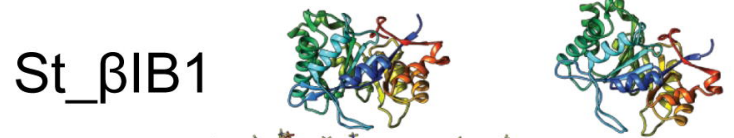
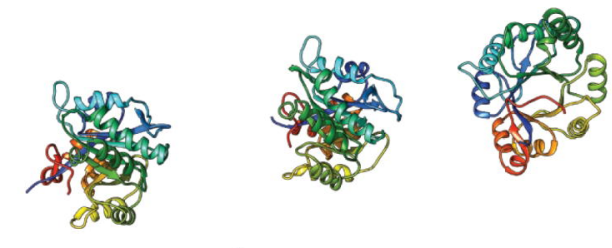

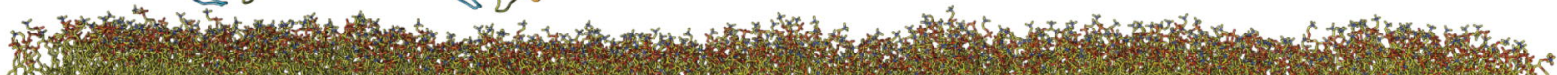
IS POPC

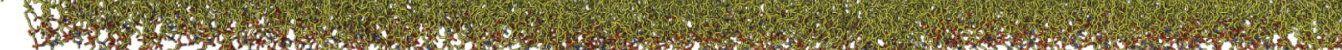

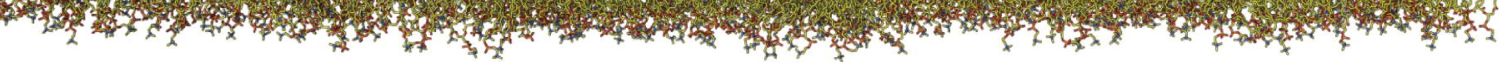
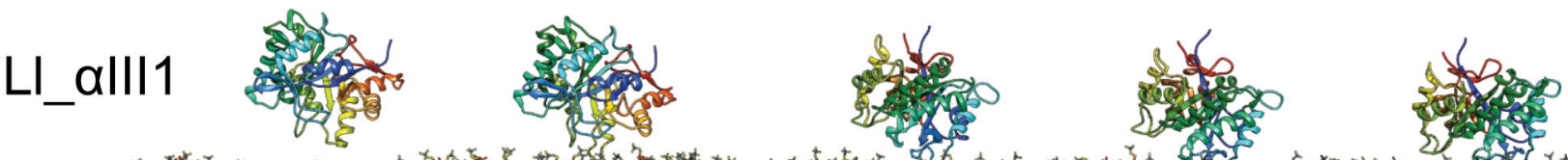

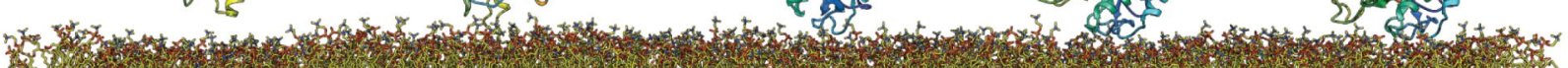
If $P O P C$ .
Li_alA1
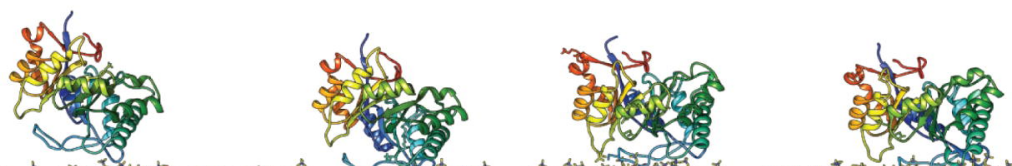

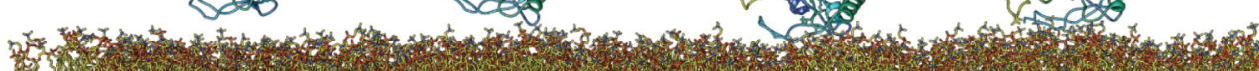

ar.

7. POPC

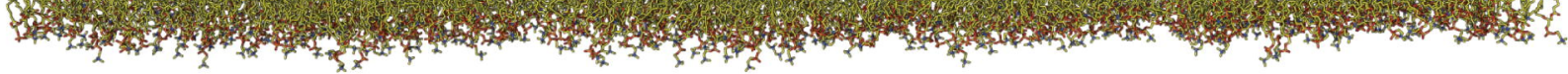

Ons $\quad 80$ ns $\quad 160$ ns 270 ns 300 ns


A)

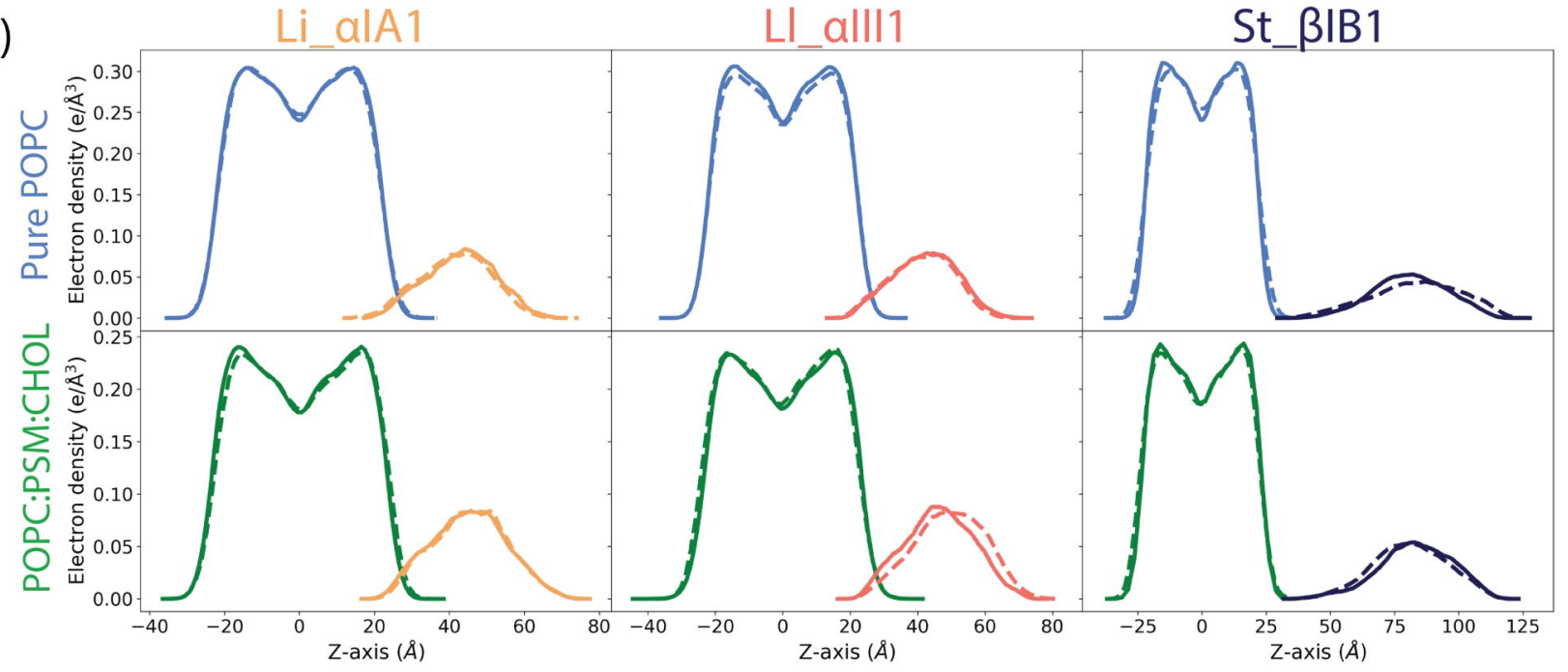

B)
C)

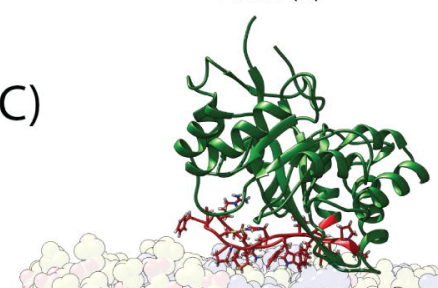

D)

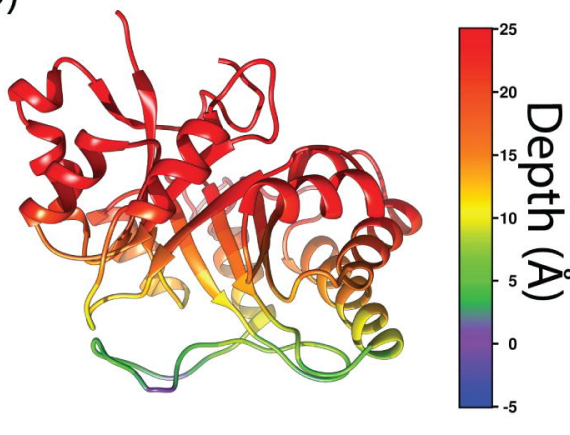




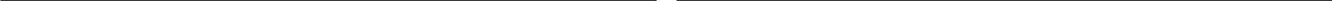




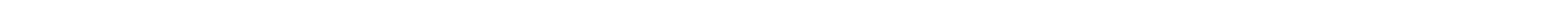



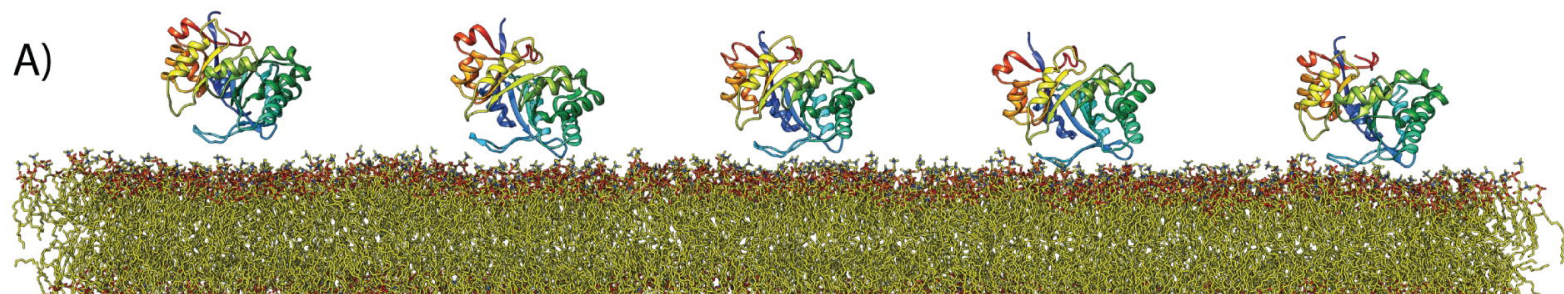

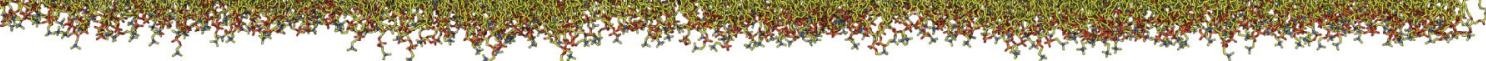

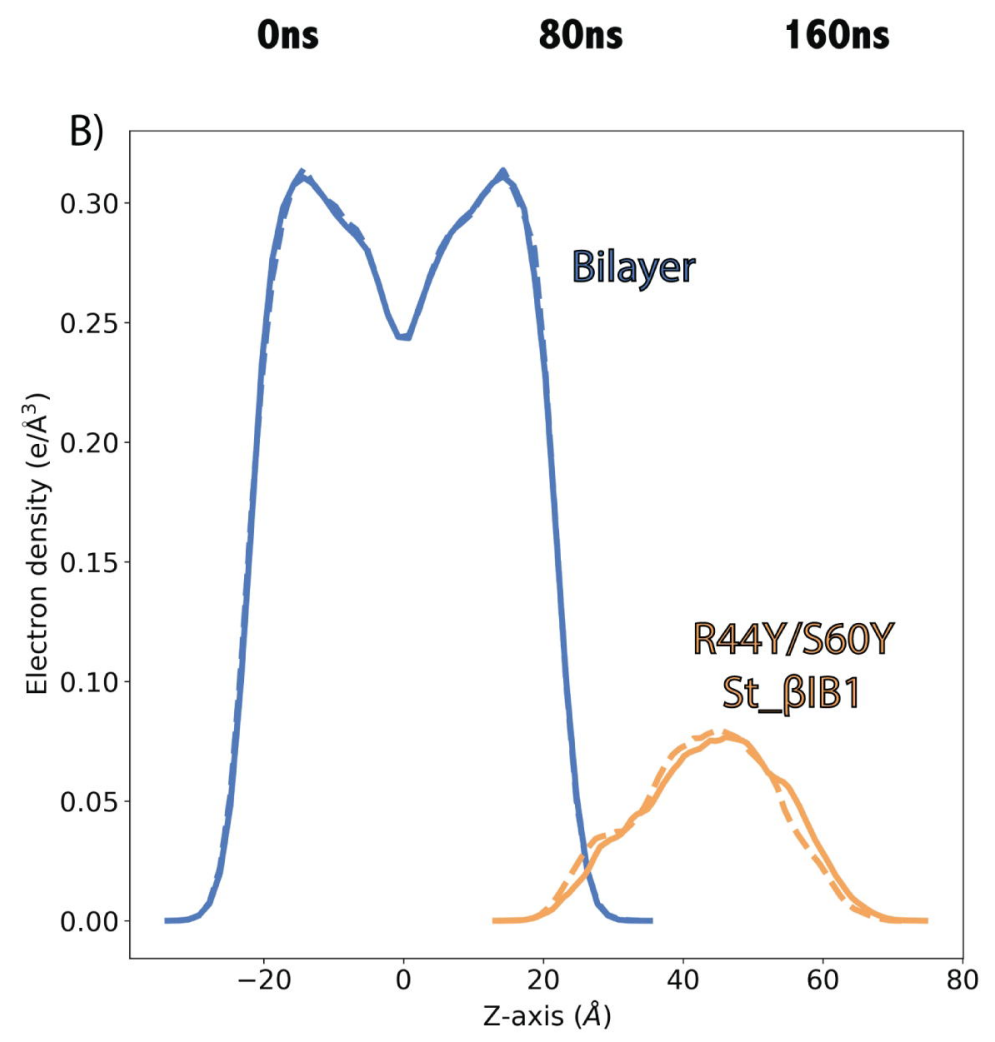





\begin{tabular}{|c|c|c|c|c|}
\hline LI_ $\alpha I I I i$ & ․ & EADVTFK-GSVPTYTYHGTPCDFGRDCIRWEY & $\cdots$ & DGLTNFS--PLGDMAR \\
\hline $\mathrm{LI}^{-} \alpha \operatorname{III2}$ & .. & EADITFK-GAVPTYSYHGTPCDFGRDCIRWEY & $\cdots$ & DGLTNWA--KLGDMAR \\
\hline $\mathrm{Li}^{-} \alpha \mathrm{IVA} 1$ & ... & TDITFDDDAMAE YS HGVPCDCRRWCHK WY & $\ldots$ & DGITNCI---LRGLDR \\
\hline $\mathrm{Li}^{-} \alpha \mathrm{V} 1$ & ... & ---VTFTKSANAE T T HGVPCDCHRWCKKWEY & $\ldots$ & DGITNCW---LRTLKR \\
\hline$i_{-}^{-} \alpha I I 1$ & $\cdots$ & EFDINFDKNGKPV TYHGVPCDCFRSCLNWEY & . . & DGITNCI---ARGLSR \\
\hline ru_ $\alpha I C 2$ & .. & ETDVSFDKQANPE $K$ THGVPCDCGRNCRY $\bigcirc \mathrm{TK}$ & $\cdots$ & DGITNCW---LRGFKR \\
\hline g_ō $I C 1$ & .. & ETDVSFDKQANPE TYHGIPCDCGRACLH TK & .. & DGITNCL---LRGLTR \\
\hline$i \_\alpha I A 2 a i$ & ... & ETDVSFDDNANPE TYHGI PCDCGRSCLKWEN & . . & DGITNCL---LRGLDR \\
\hline$i \_\alpha I A 1 a$ & $\cdots$ & ETDVSFDDNANPE TYHGIPCDCGRNCKKYEN & . & DGITNCL---PRGLSR \\
\hline$i \alpha I A 1 b i$ & ... & ETDVSFDDNANPE T HGIPCDCGRNCKKYEN & $\cdots$ & DGITNCL---PRGLSR \\
\hline $\bar{x} \alpha I B 2 b i$ & $\ldots$ & ETDVSEDSSANPE Y TYHGVPCDCRRWCKKWEY & $\ldots$ & DGITNCVASFIRGLDR \\
\hline $\bar{\alpha} I$ B3ai & ... & ETDVSFDKNANPE TYHGIPCGCGRTCTKSEK & $\ldots$ & DGITNCL---PRGLDR \\
\hline$=\alpha I A 1 i i$ & . & ETDVSFDKDANPE TYHGVPCDCGRSCLK $W Y$ & . & DGITNCL---LRGLSR \\
\hline$=\alpha$ IB1 & . & ETDVSFDKNANPE T HGIPCDCGRTCTK EN & ... & DGITNCL---LRGLSR \\
\hline$\alpha I B 1 a$ & $\cdots$ & ETDVSFDSSANPE $Y$ TYHGIPCDCGRTCTKWEN & $\cdots$ & DGITNCL---LRGLSR \\
\hline P_BIE2 $i$ & .. & ETDITFSPEGEPESA HGVPCDCKRWCDRTVS & ... & DGITNCVALLFRGTSR \\
\hline a_BIIA1 & .. & FDIDFDENGIAK THGIPCDCGRLCTKSAV & $\cdots$ & DGITSCF---ARGTDR \\
\hline SP_ $\beta$ III1 & $\cdots$ & EFDVTFDPNSKADYTYHKVPCDCGRTCGK $Y$ EV & $\cdots$ & DGITDCL---ARRSTR \\
\hline D_BIF1 & ‥ & ELDISFDSNGKAE $Y$ T YHGTPCDCF'RSCTRYES & ... & DGITNCL---NRGTDR \\
\hline Li_ßID1 & ... & ESDVSFDSNGKPE KM HGSPCDCGRSCKR MS & ... & DGITNCL---FRIMKR \\
\hline Lar_BID1 & . & ESDVSFDSDGKPE KM HGIPCDCGRKCLN MS & $\cdots$ & DGITNCI---FRVMTR \\
\hline St_BIB1 & .. & ELDVEFSKSGTAL RT THGPCDCFRSCTRSEK & ... & DGITNCL---PRDDNR \\
\hline LIAI & ... & EADLAFTSDGTADEM HGVPCDCF'RSCTRSEK & ... & DGITNCW---VRDDDR \\
\hline$\beta I A 1 i$ & ... & ELDVAFTADGTADKM HGVPCDCFRSCTRTEG & ... & DGITNCL---PRGDYR \\
\hline BIAla & ... & ELDVAFSDDGTAE KM HGVPCDCFRSCKRTET & .. & DGITNCL---PRGDYR \\
\hline
\end{tabular}

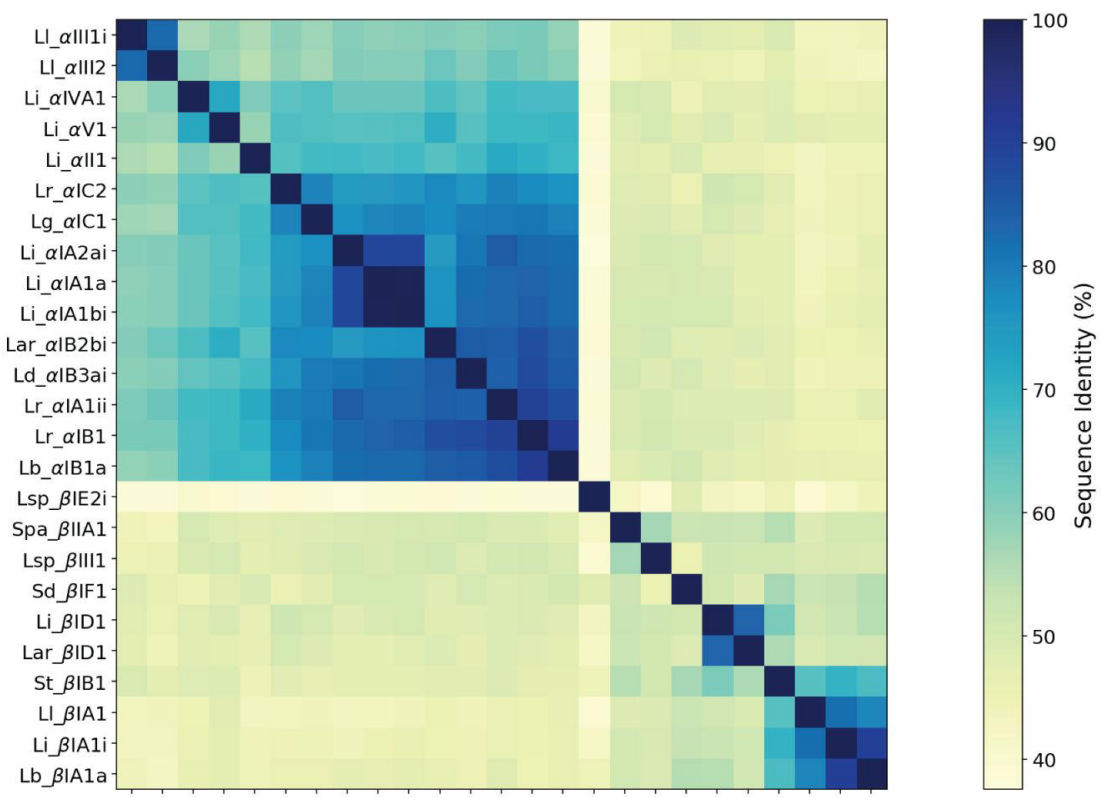

